\title{
The surface-weight illusion: On the contribution of grip force to perceived heaviness
}

\author{
GERHARD RINKENAUER, STEFAN MATTES, and ROLF ULRICH \\ University of Wuppertal, Wuppertal, Germany
}

\begin{abstract}
Previous psychophysical studies have shown that an object, lifted with a precision grip, is perceived as being heavier when its surface is smooth than when it is rough. Three experiments were conducted to assess whether this surface-weight illusion increases with object weight, as a simple fusion model suggests. Experiment 1 verified that grip force increases more steeply with object weight for smooth objects than for rough ones. In Experiment 2, subjects rated the weight of smooth and rough objects. Smooth objects were judged to be heavier than rough ones; however, this effect did not increase with object weight. Experiment 3 employed a different psychophysical method and replicated this additive effect, which argues strongly against the simple fusion model. The whole pattern of results is consistent with a weighted fusion model in which the sensation of grip force contributes only partially to the perceived heaviness of a lifted object.
\end{abstract}

The perceived heaviness of a lifted object is influenced by its surface texture. When an object is grasped between the thumb and the index finger, perceived heaviness increases with the smoothness of the object's surface texture. Flanagan, Wing, Allison, and Spenceley (1995) have recently documented this intriguing phenomenon in a psychophysical study. These authors asked their subjects, in a weight discrimination task, to lift small canisters, using a precision grip with the thumb and index finger. The test canister not only varied in weight but also in surface texture, which was either smooth or rough and, thus, more or less slippery. In each trial, the subjects compared the test canister with a reference canister and judged which one was heavier. The surface textures of the test and the reference were either identical or different. When the test canister was rough and the reference smooth, the subjects tended to underestimate the weight of the test canister. Analogously, when the test canister was smooth and the reference rough, the subjects tended to overestimate the test canister's weight. Thus, a slippery object is judged to be heavier than a rough one of identical weight.

In a further experiment, Flanagan et al. (1995) tested whether this surface-weight illusion could be attributed to the texture of the object per se or whether texture exerts only an indirect effect on perceived heaviness. In this control experiment, the subjects were asked to use a hor-

This work was supported by the Deutsche Forschungsgemeinschaft (UL 88/103) and by a seed grant of the University of Wuppertal. We thank J. Randall Flanagan, Allen Osman, and an anonymous reviewer for helpful comments. Furthermore, we thank Hiltraut Müller-Gethmann, Steffen Katzner, and Jutta Stahl for running the experiments and Jeff Miller for the loan of his ANOVA program "MrF." We also thank Rosa Maria Puca for technical support. Correspondence concerning this article should be addressed to G. Rinkenauer, General Psychology I, FB 3, University of Wuppertal, Gauss-Strasse 20, D-42097 Wuppertal, Germany (e-mail: rinkenau@uni-wuppertal.de). izontal precision grip, with the distal pad of the thumb supporting the object from below and the tip of the index finger on top. In this case, differences in grip force across textures are negligible, since the index finger needs to provide little friction to stabilize the object. Under this horizontal condition, the surface-weight illusion disappeared, indicating that the surface texture of the object is not the direct cause of this illusion. This conclusion was strengthened further in a follow-up study (Flanagan \& Wing, 1997). In this study, the subjects were asked to maintain the same level of grip force for smooth and rough surfaces, and again surface texture did not influence perceived heaviness. Both zero effects led to the conclusion that the surface-weight illusion depends only indirectly on surface texture.

More specifically, Flanagan and collaborators (Flanagan \& Wing, 1997; Flanagan et al., 1995) assumed that this illusion originated from adjusting grip force to the surface texture of the object to be lifted. It is well documented that when holding an object, using a vertical precision grip, subjects produce more grip force when its surface is smooth than when it is rough (Johansson \& Westling, 1984; for a review, see Johansson, 1996). This adjustment of grip force is necessary to prevent the object from being dropped. From a physical point of view, in order to prevent a slip when lifting an object with a vertical precision grip, a minimum grip force of $G=W /$ $(2 \cdot \mu)$ is necessary, where $W$ denotes the physical weight of the object and $\mu$ is the friction coefficient between object and skin. ${ }^{1}$ Therefore, the more slippery a surface texture (i.e., the smaller the $\mu$ ), the greater must be the minimum grip force to prevent a slip. Hence, Flanagan et al. hypothesized that, when subjects lift a slippery object, the increase in grip force, owing to the smoothness of the object's surface, induces an overestimation of its weight.

This hypothesis of a surface-weight illusion suggests that subjects somehow fail to distinguish between object 
weight and grip force when holding an object (Flanagan, 1996). Accordingly, the central nervous system (CNS) is unable to partition the sources of neuronal activation originating from grip force and object weight. Hence, both sources of activation seem to fuse, and thus, the summed activation provides the neural basis for judging object weight. This fusion hypothesis receives support from a study by Kilbreath and Gandevia (1991) in which subjects estimated a target load that acted on the digit of one hand. In another condition, a concurrent load was simultaneously acting on another digit of the same hand. The perceived heaviness of the target load increased when the concurrent load was simultaneously applied. To account for this biasing effect of the concurrent load on the perceived heaviness of the target load, Kilbreath and Gandevia assumed that the CNS is unable to separate the destination of motor commands to functionally related muscles; therefore, weight judgments about the target load are biased by the muscle activity needed to counteract the concurrent load.

This account seems also to apply to the surface-weight illusion, suggesting that the CNS cannot separate motor activity related to object weight and grip force. Consequently, slippery objects are perceived as being heavier than rough ones, because a higher amount of grip force is necessary to prevent a slip and, hence, the total level of neuronal activity to hold an object is higher. According to this fusion hypothesis, the smoothness of an object exerts only an indirect effect on perceived heaviness. The direct cause is the total amount of neuronal activity arising from both grip and lift force. The latter force corresponds to the object's weight when the object is in a neutral position. The former increases with both object weight and the slipperiness of the object's surface.

Presumably, the simplest version of this fusion hypothesis suggests that the sources of neuronal activities are summed and that this summed activity provides the neuronal basis on which perceptual processes operate in assessing the heaviness of the object. In order to model this fusion hypothesis, let $A$ be the neuronal activity associated with muscle force $F$ and assume that this activity is proportional to $F$-that is, $A=c \cdot F$. Thus, the neuronal activities resulting from lift force and grip force are $A_{W}=$ $c \cdot W$ and $A_{G}=c \cdot G$, respectively. According to this fusion model, the total neuronal activity, $A_{T}$, associated with lifting an object of weight $W$ would then be $A_{T}=A_{W}+$ $A_{G}=c \cdot(W+G) \cdot{ }^{2}$ Therefore, this simple fusion model holds that the perceived heaviness, $P$, of the lifted object is a monotonic function of the summed muscle forces $T=W+G$-that is, $P=f(T)$.

For a large class of plausible functions that map $T$ onto $P$, this fusion model predicts that the surface-weight illusion should be more pronounced for heavy objects than for light ones. ${ }^{3}$ This prediction follows because $G=W /$ $(2 \cdot \mu)$ increases with $W$ more steeply for smooth (small $\mu$ ) than for rough surfaces (large $\mu$ ). In other words, object weight is expected to have a greater impact on perceived heaviness when the object's surface is more slippery. It is the goal of this paper to assess this prediction.

Three experiments were carried out. Experiment 1 replicated the study of Johansson and Westling (1984) and was conducted to ensure that grip force increases more steeply with object load when the surface texture is smooth than when it is rough for the load ranges employed in Experiments 2 and 3. In Experiment 2, the subjects rated objects of different weights and surface textures. Interestingly, and contrary to the above prediction, an additive effect of object weight and surface texture was obtained on perceived heaviness. Experiment 3 utilized a different methodological approach to assess the generality of the conclusion arrived at in Experiment 2. Again, an additive effect of object weight and surface texture on perceived heaviness was obtained. In the General Discussion section, we will show that an elaborated version of the fusion model can account for the results obtained.

\section{EXPERIMENT 1}

In this experiment, the subjects lifted an object varying in weight (90 vs. $150 \mathrm{~g}$ ) and surface texture (smooth vs. rough). Grip force of the vertical precision grip was measured for each factorial combination. The purpose of this experiment was twofold. First, when grip force adapts to object weight, the increase of grip force with object weight is expected to be more pronounced for the smooth than for the rough surface (cf. Johansson \& Westling, 1984), according to the relation $G=W /(2 \cdot \mu)$. Hence, weight and surface texture should exert an interactive effect on grip force. Second, and more important, we wanted to ensure that such an interactive effect can be established within the 90-150 g range of object weights. The identical range was employed in Experiments 2 and 3 to assess whether object weight and surface texture yield an analogous interaction effect on perceived heaviness, as the simple fusion model would suggest. We employed this narrow range of object weights because a larger range may induce postural changes and, thus, change the pattern of the involved muscle groups when judging the weight of a light, as opposed to an especially heavy, object. Such changes might complicate the interpretation of results. Therefore we decided to restrict the range of object weights to $90-150 \mathrm{~g}$.

\section{Method}

Subjects. Seven female and 9 male students (mean age, 26.5 years) participated in this experiment. Two subjects were left-handed. The subjects were recruited from the campus of Wuppertal University. A single session lasted about $15 \mathrm{~min}$, and the subjects received $3 \mathrm{DM}$ for participating.

Apparatus and Stimuli. The object to lift was a force-sensitive manipulandum. It looked like a metal box of size $80 \times 35 \times$ $25 \mathrm{~mm}$. The width of $35 \mathrm{~mm}$ corresponded to the grip diameter. The manipulandum was suspended lengthwise from a tripod with a 
thread. The upper part of the manipulandum was covered by either a polyamide material or a coarse-grain sandpaper (240 GRIT or type 40 according to German DIN) to provide a smooth or a rough surface, respectively. The weight of the manipulandum was either 90 or $150 \mathrm{~g}$ without the weight of the thread, which was negligible and the same in both conditions. A force transducer within the box registered grip force with a resolution of $0.5 \mathrm{cN}$.

Estimation of friction coefficients. To estimate the friction coefficients, we employed the technique of the inclined plane. Five subjects were asked to lay their forearm and hand on a horizontal lever (palm up and fingers stretched). A metal plate (length $\times$ width $\times$ thickness $=65 \times 21 \times 2.5 \mathrm{~mm}$, weight $=26 \mathrm{~g}$ ) coated with sandpaper or polyamide was put lengthwise on the middle and index fingers. Then, the experimenter rotated the lever slowly downward until the metal plate began to slip. The experimenter recorded the angle of the fingers surface for this position. The tangent of the slip angle was calculated as the friction coefficient. This procedure was repeated five times for each surface texture, and the average friction coefficient across these five trials was calculated for each subject. A $t$ test on the averaged friction coefficients revealed a highly significant difference between polyamide and sandpaper $(t=6.38$, $d f=4, p<.01$ ). The average friction between skin and surface was approximately $\mu=0.48$ for the polyamide material and $\mu=1.10$ for the sandpaper.

Procedure. During the whole session, the subject was seated comfortably in a chair. The manipulandum suspended from the tripod was at chest height and was $50 \mathrm{~cm}$ in front of the subject. The subject grasped the manipulandum, using a vertical precision grip with the dominant hand. During the lift, the arm was not supported. The duration of a single lift was typically $3 \mathrm{sec}$. Grip force was recorded over an interval of $1 \mathrm{sec}$ within the second half of this period. The experimenter announced the beginning and the end of this 3-sec period. The intertrial interval between two consecutive lifts was between 15 and $30 \mathrm{sec}$. There were 10 trials per factorial combination of object weight and surface texture. The trials of each combination were blocked, and the different blocks were counterbalanced across subjects, according to a Latin square.

\section{Results and Discussion}

Mean grip force was computed for each subject and each factorial combination. Figure 1 depicts mean grip force as a function of object weight and surface texture. ${ }^{4}$ An analysis of variance (ANOVA) revealed a highly sig-

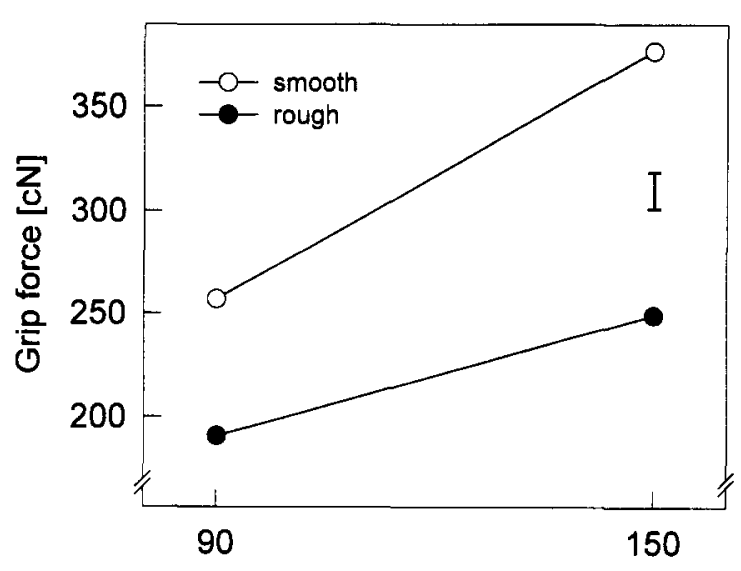

Object weight [g]

Figure 1. Mean grip force as a function of object weight and surface texture. nificant main effect of object weight $[F(1,15)=57.8, p<$ $.001]$; as expected, grip force increased with object weight. In agreement with former studies (cf. Johansson, 1996), grip force was higher for the smooth than for the rough surface texture $[F(1,15)=120.5, p<.001]$. However, theoretically more important for the purpose of this paper, there was a highly significant interaction between both factors $[F(1,15)=49.3, p<.001]$; consistent with the predictions outlined above, object weight had a stronger effect on grip force for smooth than for rough surface textures. The next two experiments were conducted to assess whether an analogous interaction emerges for perceived heaviness, as the simple fusion hypothesis would suggest.

\section{EXPERIMENT 2}

Experiment 2 employed a rating procedure to assess the effects of object weight and surface texture on perceived heaviness. This procedure was quite similar to a psychophysical method employed by Anderson (1970) for testing an additive model of the so-called size-weight illusion. In short, in each trial, the subjects first lifted a standard cylinder, then a test cylinder, and rated the weight of the test cylinder on a scale ranging from 0 (lightest) to 20 (heaviest). The standard weight served as a point of reference and corresponded to the value 10 on the rating scale. The weight of the test cylinder was $90,100,110$, $120,130,140$, or $150 \mathrm{~g}$, and the surface was either smooth or rough, whereas the weight of the standard remained constant ( $120 \mathrm{~g}$ ) and its surface (cotton, $\mu=0.74$ ) was of intermediate smoothness. According to the fusion hypothesis, we expected that the weight of the test cylinder would have a more pronounced effect on judged heaviness for smooth than on that for rough surfaces.

We also employed a horizontal precision grip condition to validate the rating procedure. According to Flanagan et al. (1995), the surface-weight illusion should disappear in this horizontal grip condition, if the illusion depends on grip force and not on surface texture per se.

\section{Method}

Subjects. Twenty-five female and 21 male subjects (mean age, 27.3 years) participated in a single session lasting about $1 \mathrm{~h}$. Five subjects claimed to be left-handed. All the subjects were naive about the purpose of this study and received $10 \mathrm{DM}$ for their participation.

Apparatus and Stimuli. The test cylinders were plastic canisters, which were $55 \mathrm{~mm}$ long and $41 \mathrm{~mm}$ in diameter. The weight of each cylinder was adjusted by variable brass disks that were fixed inside the cylinder. As in Experiment 1, the plastic canisters were covered by either a polyamide material or a coarse-grain sandpaper. In the vertical condition, the experimenter presented the standard and test cylinders on a carriage. In the horizontal condition, cylinders were put horizontally on a fixture, which was assembled on the carriage to permit a comfortable horizontal grasp. The experimenter sat behind a partition (Venetian blind), so that the experimenter could watch the subject, yet the subject could not see the experimenter, in order to avoid distraction.

Procedure and Experimental Task. Subjects were seated comfortably in front of a table. The two grip conditions were blocked, and each block consisted of a practice part followed by an experi- 
mental part. During the practice part, only cylinders with intermediate smoothness (cotton surface) were used, and each of the seven test cylinders was presented twice. During the experimental part, the seven objects with a rough surface and the seven objects with a smooth surface were each presented four times. The order in which the test cylinders were presented was randomized. The order of the two grip conditions was balanced over subjects. Each grip condition started with the presentation of two end-anchors of 70 and $170 \mathrm{~g}$. These were lifted several times and compared with the standard, and subjects were told that these weights corresponded to the minimum and the maximum on the rating scale, respectively.

At the beginning of each trial, the experimenter placed the standard cylinder and one of the test cylinders on the carriage and positioned it in front of the subject. The subject first lifted the standard cylinder and then the test cylinder, using a precision grip with the preferred hand. The subject's task was to tell the experimenter how heavy the test cylinder felt by expressing it verbally on the rating scale between 0 and 20 .

Design. The experiment factorially combined the three withinsubjects factors of texture (smooth vs. rough), weight of test cylinder $(90,100,110,120,130,140$, and $150 \mathrm{~g})$, and grip condition (vertical vs. horizontal). The dependent variable was rated heavinessthat is, the mean heaviness rating for each subject in each of the 28 factorial combinations.
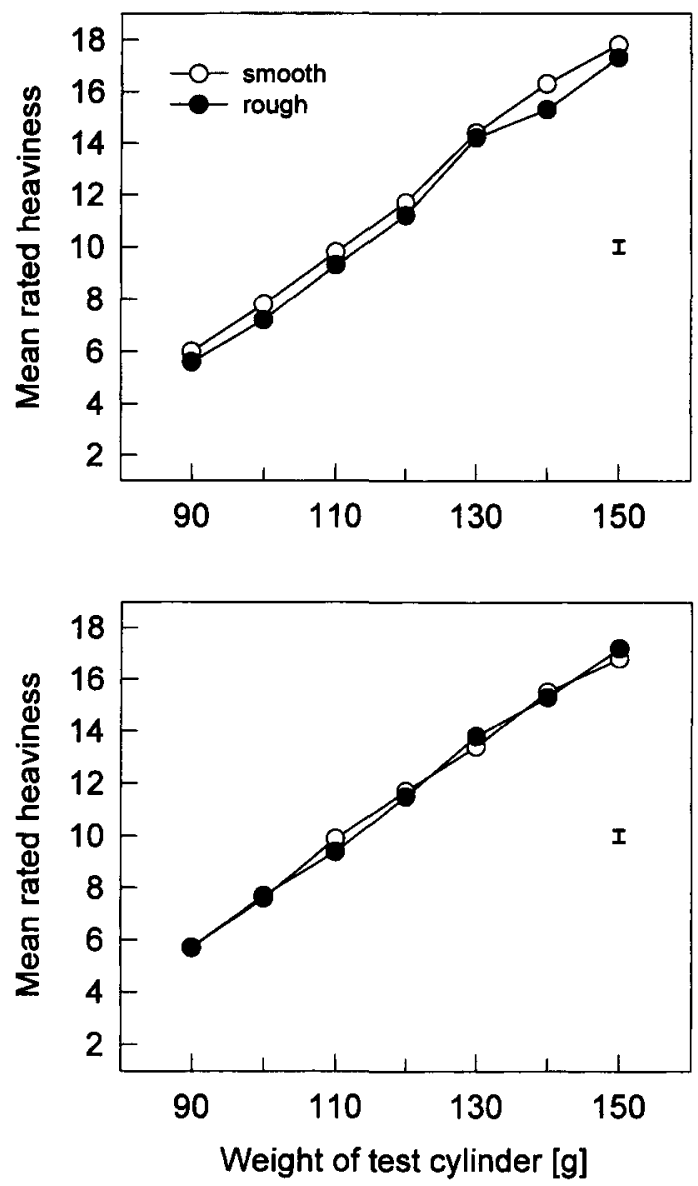

Figure 2. Mean rated heaviness as a function of test cylinder weight and surface texture. Upper panel, vertical grip; lower panel, horizontal grip.

\section{Results and Discussion}

Figure 2 shows the mean heaviness rating as a function of object weight and surface texture. The upper panel depicts the results for vertical grips, and the lower panel for horizontal ones. An ANOVA on mean ratings was conducted, with the factors being texture, weight, and grip condition. As was to be expected, perceived heaviness increased with object weight $[F(6,270)=$ $748.6, p<.001]$ and was not influenced by grip condition $[F(1,45)=1.2, p=.29]$. The increase of mean rated heaviness with object weight was slightly steeper for vertical than for horizontal grips $[F(6,270)=3.1, p<.01]$. An additional regression analysis showed that the slope for the vertical condition was significantly larger than the slope for the horizontal one [slopes, .202 vs. .195; $F(1,45)=4.8, p<.05]$. This slope difference suggests that the subjects were more sensitive to weight changes for vertical precision grips than to those for horizontal ones.

As in the study of Flanagan et al. (1995), the subjects judged smooth objects to be heavier than rough ones $[F(1,45)=7.4, p<.01]$. Furthermore, and consistent with this previous study, surface texture influenced judged heaviness only in the vertical but not in the horizontal grip condition $[F(1,45)=11.0, p<.01]$; for vertical grips, the mean ratings were 12.0 for smooth and 11.5 for rough surfaces. The respective means were 11.6 and 11.5 for horizontal grips. Thus, the present experiment replicated fully the surface-weight illusion for vertical grips as reported by Flanagan et al. No further effects were statistically significant.

The main theoretical issue of this experiment was to assess whether the surface-weight illusion increases with object weight in the vertical condition, as one would expect on the basis of the simple fusion model. However, Figure 2 does not indicate such an interaction. A separate ANOVA for the vertical grip condition again yielded highly significant effects of object weight $[F(6,270)=$ $543.3, p<.001]$ and of surface texture $[F(1,45)=14.1$, $p<.001]$. However, the interaction of both factors was not significant $[F(6,270)=1.0, p=.43]$, suggesting an additive effect of both factors on perceived heaviness. In conclusion, then, the present results indicate, contrary to our expectation, that the surface-weight illusion does not become more pronounced as object weight increases.

\section{EXPERIMENT 3}

Experiment 3 used a different psychophysical method to assess the generality of the results found in the preceding experiment. Specifically, we sought to examine whether the theoretically unexpected additive effect of surface texture and object weight can also be obtained with a different psychophysical approach, because one might argue that this result could reflect an artifact of the method employed in Experiment 2 (see, e.g., Sarris \& Heineken, 1976). 
In the present experiment, the subjects lifted a standard weight and a comparison weight in each trial and judged whether the latter appeared lighter or heavier than the former. Standard weight ( 90 vs. $150 \mathrm{~g}$ ) and surface texture (smooth vs. rough) of the comparison were factorially combined. For each factorial combination, the point of subjective equality (PSE) was estimated. Shifts in PSE were assumed to indicate differences in perceived heaviness. According to the simple fusion hypothesis, the PSE difference for smooth and rough objects should increase with standard weight. If, however, surface texture and weight again fail to show such an interaction, this would provide further evidence against the notion that the surface weight illusion depends on object weight.

\section{Method}

Subjects. Nine female and 9 male subjects (mean age, 25.1 years) participated in a single session lasting about $1 \mathrm{~h}$. Two subjects claimed to be left-handed. All the subjects were naive about the purpose of this study and received $10 \mathrm{DM}$ for their participation.

Apparatus and Stimuli. A wooden box (length, $500 \mathrm{~mm}$; height and depth, each $150 \mathrm{~mm}$ ) lay crosswise in front of the subject on a table. Four cylinders, as were described in Experiment 2, were placed on this box side by side, with a distance of $100 \mathrm{~mm}$ from center to center. As in Experiment 1, the subject and the experimenter were separated by a partition. The experimenter sat on the other side of the table behind the partition and could move the box from left to right in such a way that the subject always reached to the same object location. The partition guaranteed that the subject saw none of the experimenter's activities. An arrow attached to the partition pointed downward to the location of the cylinder to be lifted.

The experimenter moved the box so that the cylinder to be lifted was always located under the arrow. The leftmost cylinder was covered with coarse-grain sandpaper, and the rightmost cylinder with polyamide material. Both intermediate cylinders were covered with cotton and served as standard weights. Each of the four cylinders had a stiff thread attached to the bottom that led through a hole ( $8 \mathrm{~mm}$ in diameter) in the wooden box. The rear side of the box was open, and thus, the experimenter could hang weights on the threads. The length of the thread was such that the weight reached exactly to the floor of the wooden box, which was covered with a thin layer of foam. Thus, no jerk could be perceived when the cylinder on top of the box was lifted.

The experimenter was provided with a set of weights ranging from 20 to $250 \mathrm{~g}$, in steps of $5 \mathrm{~g} .{ }^{5}$ The weight of the two intermediate cylinders with the cotton surface was 90 and $150 \mathrm{~g}$.

The subjects pressed one of two keys on the computer keyboard, which were labeled lighter and heavier, to indicate how they perceived the second weight when compared with the first one. The keyboard was placed in front of the subject, and the judgments were recorded by a microcomputer. The monitor of the computer was on the side of the experimenter and, thus, could not be viewed from the subject's position.

Procedure and Experimental Task. The total number of trials was 160 , and the temporal course of a single trial was as follows. The experimenter moved the wooden box in such a way that one of the two standard weights ( 90 or $150 \mathrm{~g}$, covered with cotton) was below the arrow. The subject lifted the weight about $10 \mathrm{~mm}$ and put it back after about $2 \mathrm{sec}$. Then the experimenter moved the box, and the subject lifted one of the two comparison weights (smooth or rough) and pressed the key labeled lighter if the comparison appeared lighter than the standard and the one labeled heavier if the comparison appeared heavier. The subjects were encouraged to choose a response category when they perceived the two weights to be equal (forced choice). The experimenter removed the weight from the comparison and prepared the device for the next trial by attaching a weight to one of the two comparisons. This change of weights produced an intertrial interval of about $5 \mathrm{sec}$. The start of the next trial was signaled by the movement of the box to one of the two standards. Each of the four conditions $(90 \mathrm{~g} / \mathrm{smooth}, 90 \mathrm{~g} / \mathrm{rough}, 150 \mathrm{~g} / \mathrm{smooth}$, and 150 $\mathrm{g} /$ rough) was employed 40 times, in a randomized order.

The variable comparison weight was changed according to an adaptive rule (Kaernbach, 1991). For each condition, two separate adaptive percentile estimations were used (25th and 75 th percentile) - that is, this adaptive rule determined the comparison weights where the response heavier was given with the probability .25 and .75 , respectively. For each condition, the initial comparison weight was $40 \mathrm{~g}$ below the standard weight for the 25 th percentile and $40 \mathrm{~g}$ above the standard for the 75 th percentile. For the 25 th percentile, the comparison weight was increased by $10 \mathrm{~g}$ when the subject judged the comparison to be lighter than the standard and decreased by $30 \mathrm{~g}$ when the subject judged the comparison to be heavier. These step sizes were reversed for the 75 th percentile. The step size of each percentile estimation was reduced by $50 \%$ when the response had changed twice from heavier to lighter or vice versa. The trials before these step size reductions were considered practice and were excluded from data analysis. All conditions were randomly mixed, and the order of presentation was computer controlled.

Estimation of the point of subjective equality. A maximum likelihood procedure was used to estimate the PSE for each combination of texture and standard weight. To this end, a logistic psychometric function (cf. Bush, 1963),

$$
\left.\operatorname{Prob} \text { ("heavier" } \mid W_{i}\right)=\frac{1}{1+e^{\left(\mathrm{PSE}-W_{i}\right) /(0.91 \cdot \mathrm{DL})}},
$$

was employed, which associates the comparison weight $W_{i}$ in the $i$ th trial with the probability of the response heavier. The parameter DL denotes the difference limen and represents the steepness of the psychometric function. The estimates of PSE and DL were those values that maximized the corresponding likelihood function. A similar procedure with stimulus selection according to a nonparametric adaptive rule and final parameter estimation by a maximum likelihood procedure has been called a hybrid procedure by Hall (1981), who discusses its advantages.

Design. Two within-subjects factors were factorially combined: standard weight (90 vs. $150 \mathrm{~g}$ ) and surface texture (smooth vs.

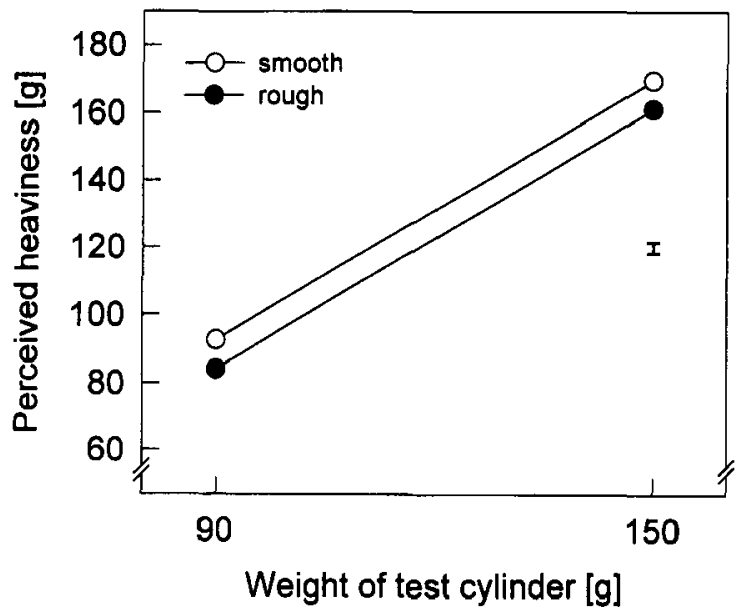

Figure 3. Perceived heaviness as a function of test cylinder weight and surface texture. 
rough). The dependent variable was PSE for each subject and each factorial combination.

\section{Results and Discussion}

An ANOVA, with texture and standard weight as factors, was performed for PSE. Figure 3 reports perceived heaviness, as estimated from the PSEs, as a function of surface texture and standard weight. ${ }^{6}$ As can be seen, the two main findings of Experiment 2 were replicated. First, objects with a smooth surface were perceived again as being heavier than objects with a rough surface $[F(1,17)=$ $22.1, p<.001]$; on the average, smooth objects were perceived to be $8.5 \mathrm{~g}$ heavier than rough ones. Second, and contrary to the predictions of the simple fusion model, this illusion again did not increase with standard weight $(F<1)$; the surface effect on the PSE was $8.6 \mathrm{~g}$ for the 90 $\mathrm{g}$ standard and $8.4 \mathrm{~g}$ for the $150 \mathrm{~g}$ standard. As was to be expected, the main effect of the standard weight factor was again highly significant $[F(1,17)=430.7, p<.001]$. In conclusion, then, this experiment replicated the crucial results found in Experiment $2 .^{7}$

\section{GENERAL DISCUSSION}

In this paper, we have sought to examine whether the surface-weight illusion for a vertical precision grip (with the thumb and index finger on either side of the lifted object) is more pronounced for heavy than for light objects, as a simple fusion model suggests. However, contrary to this expectation, the present results show that object weight does not influence the magnitude of this illusion; therefore, the present results argue strongly against the validity of the simple fusion model. In the following, we discuss how an elaboration of the fusion hypothesis can be reconciled with the present results. Before doing so, we shall briefly review the present main findings and the reasons why we expected that the illusion should increase with object weight.

Experiment 1 was performed to ensure that grip force actually increases more steeply with object weight for smooth than with that for rough surface textures and with the load ranges employed in the psychophysical Experiments 2 and 3 . As had been reported previously (Johansson, 1996; Johansson \& Westling, 1984), the subjects produced more grip force for slippery than for rough surface textures. Most important, however, Experiment 1 revealed a steeper increase of grip force with object weight for the slippery than with that for the rough object surface. This interaction clearly supports the idea that grip force, $G$, is adjusted according to the multiplicative relation $G=W /$ $(2 \cdot \mu)$ of object weight, $W$, and the friction between skin and object surface $(\mu)$.

Experiments 2 and 3 employed different psychophysical methods to evaluate whether an analogous interaction of object weight and surface texture can be observed for perceived heaviness, as is implied by the simple fusion hypothesis. According to this hypothesis, the surface-weight illusion arises because the CNS can- not discriminate between the sources of neural activity produced by grip force $G$ and the force $W$ for holding the object - that is, the lift force. As was explained in the introduction, if the two sources cannot be discriminated, they should somehow unify into a single neuronal code that provides the basis for the perception of object heaviness. To express this idea more quantitatively, it seemed reasonable to assume, at least as a first approximation, that the strength of this code is proportional to the sum of total forces, $T=G+W$, required to hold an object. This simple additive model predicts that perceived heaviness should increase more steeply with object weight for slippery than with that for rough objects- that is, the surfaceweight illusion becomes more pronounced as object weight increases. Experiments 2 and 3 tested this prediction.

In Experiment 2, the subjects rated objects of different weights and surface textures. Experiment 3 used a different psychophysical approach to assess whether the results in Experiment 2 depended on the particular rating method employed (Sarris \& Heineken, 1976). However, Experiment 3 replicated the results of Experiment 2, which strongly suggests that the results obtained are not due to the particular psychophysical methods employed. Both experiments replicated the surface-weight illusion reported previously by Flanagan and collaborators (Flanagan \& Wing, 1997; Flanagan et al., 1995). The subjects rated smooth objects as being heavier than rough objects of the same weight. The comparison of vertical and horizontal conditions in the study of Flanagan et al. and in Experiment 2 of the present study, as well as a control condition with fixed grip force in the study of Flanagan and Wing, strongly suggests that the surface-weight illusion is due to differences in grip force. However, as Flanagan and Wing discuss, it cannot be ruled out entirely that sensory factors may also contribute to this illusion.

The present experiments revealed that the magnitude of the surface-weight illusion did not increase with object weight, which is at odds with the predictions of the simple fusion model. However, an elaboration of the fusion model may provide a possible basis on which to account for the present results. This model elaboration is based on two assumptions. First, it abolishes the assumption of the simple fusion model that the neuronal activation associated with $W$ and $G$ is integrated in an unweighted fashion into a single perceptual code. Instead it is assumed that perceived heaviness reflects the weighted sensations of $G$ and $W$. Second, the psychophysical transformation from force to its sensation can be approximated by a $\log$ function that resembles Fechner's law. Thus, the sensation associated with grip force is equal to $S_{G}=a$. $\log (G)+c_{G}$, whereas the sensation associated with object weight is equal to $S_{W}=b \cdot \log (W)+c_{W} \cdot{ }^{8}$ Therefore, the perceived heaviness $P$ is

$$
P=S_{G}+S_{W}=a \cdot \log (G)+b \cdot \log (W)+c,
$$

where the constant $c$ is equal to the sum $c_{G}+c_{W}$. The constants $a$ and $b$ denote the weighting factors. If $G$ is replaced by $W /(2 \cdot \mu)$, it can be shown that this weighted 
Unweighted
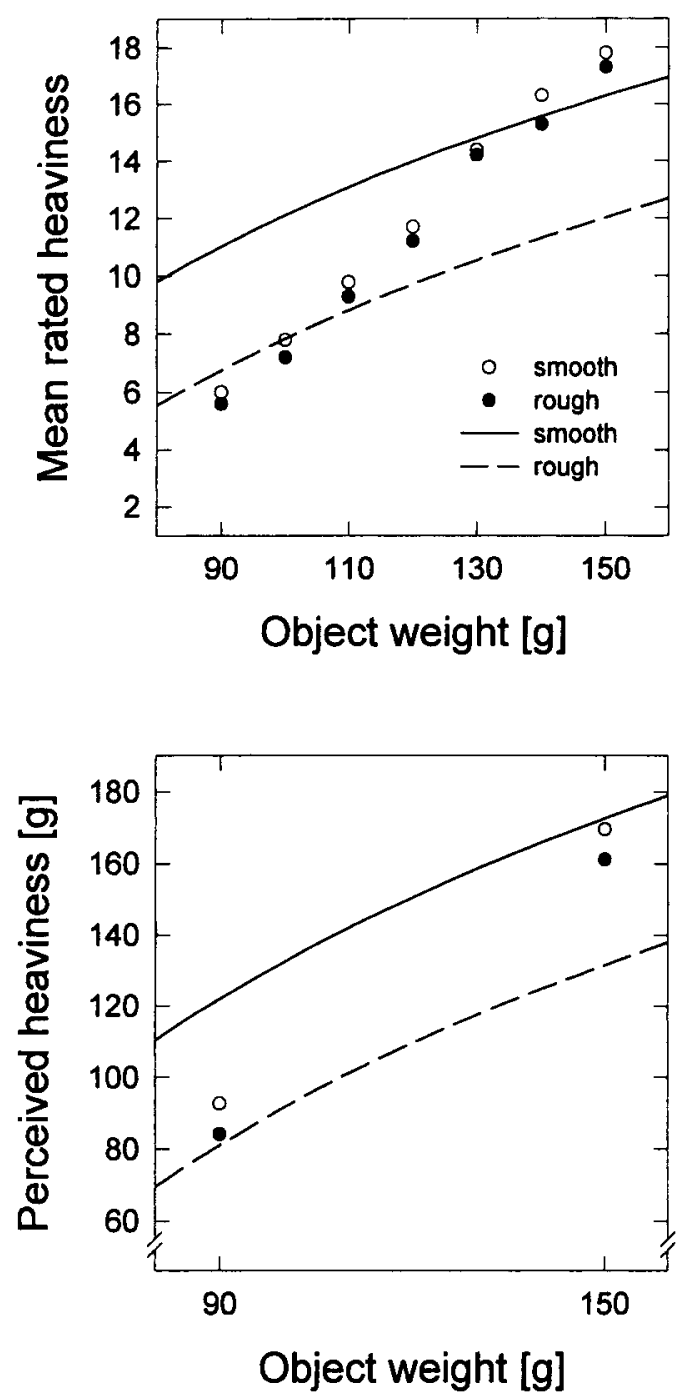

Weighted
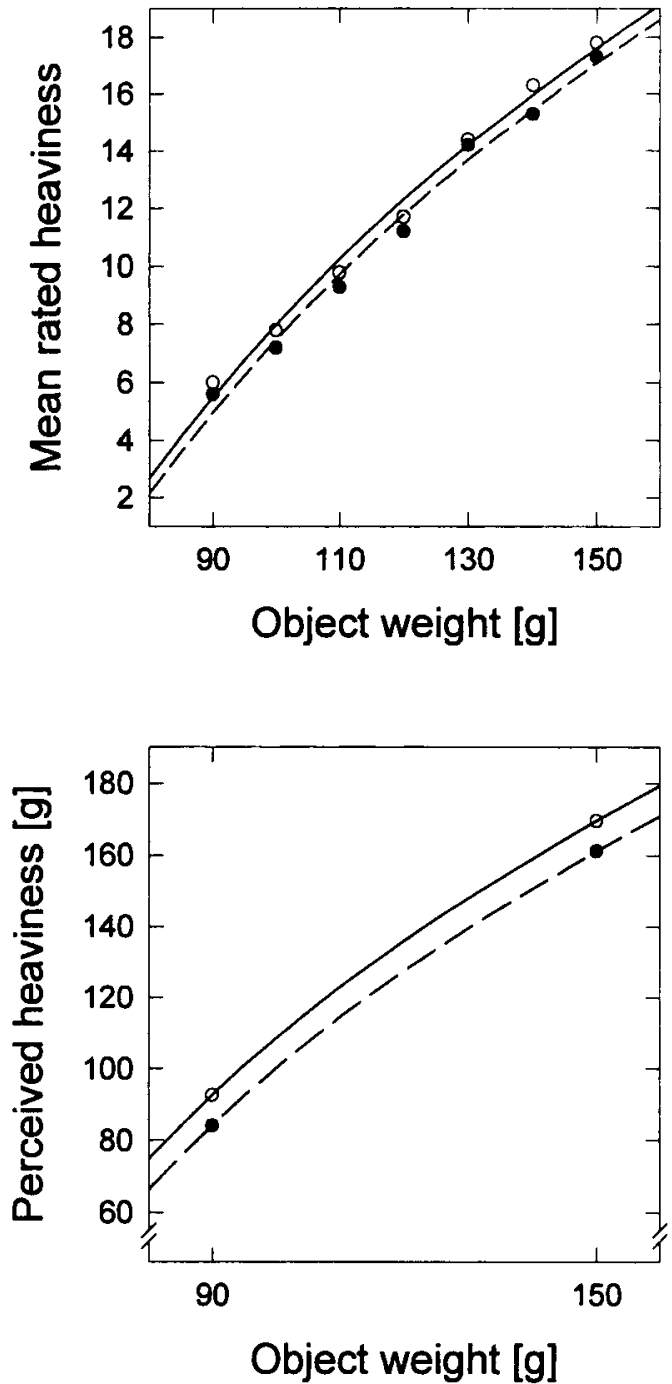

Figure 4. Predictions of the elaborated fusion model. The two lines in each panel denote the model predictions (least squares fits). Left panels, predictions if weighting factors $a$ and $b$ are equal; right panels, predictions for unequal weighting factors; upper panels, results of Experiment 2; lower panels, results of Experiment 3.

fusion model predicts an additive effect of surface texture and object weight on perceived heaviness $P .^{9}$

Figure 4 shows the predictions of the weighted fusion model for the results of Experiments 2 and 3. The left two panels show the predictions for the model under the assumption that both weights are equal, as one would expect when both sensations (i.e., $S_{W}$ and $S_{G}$ ) were equally weighted to form perceived heaviness. As can be seen, in this case the model's predictions are bad. The root mean squared error of Experiment 2 (left upper panel) and Experiment 3 (left lower panel) is 3.1 and 24.2 , respectively. In particular, the model predicts too large an effect of surface texture on perceived heaviness.

The right two panels depict the predictions of the model under the assumption that perceived heaviness emerges from a weighted sum of $S_{W}$ and $S_{G}$. In this case, the predictions are satisfactory. The root mean squared error of Experiment 2 (right upper panel) and Experiment 3 (right lower panel) is 0.4 and 0.05 , respectively. The estimated parameters for Experiment 2 are $a=1.5$, $b=53.2$, and $c=-101$, and for Experiment 3, these are $a=24, b=323$, and $c=-586$. The estimated parameters suggest that grip force contributes to a much lesser extent to perceived heaviness than lift force does because $a$ is always much smaller than $b$.

This elaborated fusion model with unequal weights suggests that the brain discriminates between the neuronal activation exerted by grip and that exerted by lift force. Proceeding from this conclusion, it seems natural to ask why the brain weights both sensations rather than relying 
solely on the sensation of lift force. One possible answer to this question is that the integration of neuronal activation emerging from grip force improves the accuracy of weight judgments. Some evidence for this conclusion stems from the study of Flanagan et al. (1995). In their second experiment, the subjects performed vertical and also horizontal precision grips. The psychometric functions for vertical grips were steeper than those for horizontal ones. This is consistent with the view that grip force improves the sensitivity of weight perception. A similar conclusion applies to the present finding of Experiment 2 that perceived heaviness increased less steeply with object weight in the horizontal grip condition. According to the weighted fusion model, the contribution of grip force would be theoretically zero in the horizontal condition, and this would reduce the slope of the perceived heaviness function.

In conclusion, then, the present study replicated the finding that the force exerted to grasp an object influences its perceived heaviness. This influence cannot be explained with a simple fusion model. Instead, the observed additivity of object weight and surface texture favors a weighted fusion model based on Fechner's law. According to this weighted model, it seems plausible that the brain incorporates grip force to increase the sensitivity of weight judgments. Further research is needed to test this functional interpretation of the weighted fusion model.

\section{REFERENCES}

ANDERSON, N. H. (1970). Averaging model applied to the size-weight illusion. Perception \& Psychophysics, 8, 1-4.

Bush, R. R. (1963). Estimation and evaluation. In R. D. Luce, R. R. Bush, \& E. Galanter (Eds.), Handbook of mathematical psychology (Vol. 1, pp. 429-469). New York: Wiley.

FlanaGAN, J. R. (1996). Action-perception coupling in judgments of hand-held loads. In A. M. Wing, P. Haggard, \& J. R. Flanagan (Eds.), Hand and brain: Neurophysiology and psychology of hand movement (pp. 415-430). New York: Academic Press.

FlanaGan, J. R., \& Wing, A. M. (1997). Effects of surface texture and grip force on the discrimination of hand-held loads. Perception \& Psychophysics, 59, 111-118.

Flanagan, J. R., Wing, A. M., Allison, S., \& Spenceley, A. (1995). Effects of surface texture on weight perception when lifting objects with a precision grip. Perception \& Psychophysics, 57, 282-290.

HALL, J. L. (1981). Hybrid adaptive procedure for estimation of psychometric functions. Journal of the Acoustical Society of America, 69, 1763-1769.

JOHANSSON, R. S. (1996). Sensory control of dexterous manipulation in humans. In A. M. Wing, P. Haggard, \& J. R. Flanagan (Eds.), Hand and brain: Neurophysiology and psychology of hand movement (pp. 381-414). New York: Academic Press.

JohansSON, R. S., \& WeSTLING, G. (1984). Roles of glabrous skin receptors and sensorimotor memory in automatic control of precision grip when lifting rougher or more slippery objects. Experimental Brain Research, 56, 550-564.

KAERNBACH, C. (1991). Simple adaptive testing with the weighted updown method. Perception \& Psychophysics, 49, 227-229.

KEBECK, G. (1994). Wahrnehmung: Theorien, Methoden und Forschungsergebnisse der Wahrnehmungspsychologie [Perception: Theories, methods, and research results of perceptual psychology]. Weinheim: Juventa Verlag.
Kilbreath, S. L., \& Gandevia, S. C. (1991). Independent digit control: Failure to partition perceived heaviness of weights lifted by digits of the human hand. Journal of Physiology, 442, 585-599.

LofTUs, G. R., \& MAsson, M. E. J. (1994). Using confidence intervals in within-subject designs. Psychonomic Bulletin \& Review, 1, 476-490. SARRIS, V., \& HEINEKEN, E. (1976). An experimental test of two mathematical models applied to the size-weight illusion. Journal of Experimental Psychology: Human Perception \& Performance, 2, 295-298. Stevens, S. S. (1975). Psychophysics: Introduction to its perceptual, neural, and social prospects. New York: Wiley.

\section{NOTES}

1. It should be noted that the minimum grip force to prevent a slip is $G_{\min }=W /(2 \cdot \mu)$. However, subjects always employ a grip force safety margin that (usually) increases with object weight (Johansson \& Westling, 1984). Thus, a more realistic equation for grip force would be $G=$ $G_{\min }+m \cdot G_{\min }=(1+m) /(2 \cdot \mu) \cdot W$, where $m$ is a positive constant Therefore, $G$ can be written as $G=W /\left(2 \cdot \mu^{*}\right)$ with $\mu^{*}=\mu /(1+m)$. This denotation of $G$ is formally equivalent to the equation for $G_{\min }$ Therefore, for sake of simplicity, we will employ $G=W /(2 \cdot \mu)$ in the main text without loss of generality.

2. This simple fusion model assumes equal weighting of $W$ and $G$. In a more realistic model, however, these weights may differ. Inasmuch as the conclusions are the same for the general and the specific versions of the fusion model, we will stick with the simple version without loss of generality.

3. For example, it is well known that, for magnitude estimates, the relation between $T$ and $P$ can be adequately described by Stevens's power law, $P=a \cdot T^{b}$, where the exponent $b$ depends on the sensory modality and $a$ is a constant (Stevens, 1975). Thus, if one proceeds from a power law relation between $T$ and $P$, the surface-weight illusion is expected to increase with object weight, because $P=a \cdot(G+W)^{b}=a \cdot[W /$ $(2 \cdot \mu)+W]^{b}=a \cdot W^{b} \cdot[1+1 /(2 \cdot \mu)]^{b}$, showing that $W$ and $\mu$ exert a multiplicative effect on $P$.

4. The error bar in this and the following figures indicates the standard error of means as estimated from the $M S_{\mathrm{e}}$ error terms of the repeated measures ANOVA (Loftus \& Masson, 1994).

5. This and all following information about weights refers to the total weight of the cylinder, the thread, and the attached weight.

6. This transformation from PSE to perceived heaviness was performed because low PSE values imply a higher perceived heaviness. To ease the visual interpretation and comparison with Experiment 2, we estimated perceived heaviness as Standard + (Standard - PSE). As an example, assume a standard of $150 \mathrm{~g}$ and a comparison with a smooth surface. Furthermore, assume that, in order to perceive the standard and the comparison as being equally heavy, the weight of the comparison has to be adjusted to $140 \mathrm{~g}$ (= PSE). This bias of $10 \mathrm{~g}$ expresses the overestimation of the comparison because of its smooth surface. Thus, a comparison of $150 \mathrm{~g}$ would be perceived as $160 \mathrm{~g}$. It should be stressed that this transformation does not affect the conclusions, yet provides a more convenient way to present the results.

7. The estimated values of DL did not vary with surface texture (DL $=7.5 \mathrm{~g}$ for smooth and $7.7 \mathrm{~g}$ for rough objects). These values are close to those obtained by Flanagan et al. (1995), as estimated from their Figure 5, upper panel.

8. The constants $c_{G}$ and $c_{W}$ reflect threshold values. More specifically, a threshold value $O$ can be computed by taking the antilog of $c=a$. $\log (O)$, where the unit of measurement for $O$ is gram (for the rationale of this antilog transformation to estimate $O$, see, e.g., Kebeck, 1994).

9. It can be shown that object weight and surface texture $\mu$ exert an additive effect on perceived heaviness: $P=S_{G}+S_{W}=a \cdot \log (G)+$ $b \cdot \log (W)+c=a \cdot \log [W /(2 \cdot \mu)]+b \cdot \log (W)+c=(a+b) \cdot$ $\log (W)-a \cdot \log (2 \cdot \mu)+c=f(W)+g(\mu)+c$.

(Manuscript received July 14, 1997; revision accepted for publication December 26, 1997.) 